\title{
Modelling photovoltaic power
}

\author{
Fotis Mavromatakis $^{1}$, Frank Vignola ${ }^{2}$ and Yannis Franghiadakis ${ }^{1}$
}

\begin{abstract}
There are several reasons why a robust and reliable model describing the instantaneous power produced by a photovoltaic system is useful. Module failures, inverter malfunction and shadowing are some of the problems leading to energy loss that can be detected with the aid of a sufficiently good model. Furthermore, a reliable model enables an investor to accurately estimate the system energy production, payback times etc. The model that has been developed adopts the known dependence upon solar irradiance and temperature but it also takes into account the airmass, the angle of incidence and the efficiency drop due to low values of solar irradiance. In addition, ohmic losses, soiling can be taken into account either as a fixed term or variable term if sufficient data are available. Generic functions describing the airmass and angle of incidence effects can be used based upon the module technology but these functions can also be determined for the specific modules under study.

The model is validated against high quality data available from the National Renewable Energy Laboratory (USA). The data consist of current-voltage traces of specific modules of different technologies along with solar irradiance at the plane of the module and module temperature. Initial results of the model validation are presented in this work. The dependence of the residuals upon solar irradiance temperature, airmass and angle of incidence is also explored.
\end{abstract}

Keywords: Photovoltaic, power, model, analysis, angle of incidence, airmass, low irradiance efficiency

\section{Acknowledgement}

This project is implemented through the Operational Program "Education and Lifelong Learning", Action Archimedes III and is co-financed by the European Union (European Social Fund) and Greek national funds

\footnotetext{
${ }^{1}$ Laboratory of Energy and Photvoltaic Systems, Department of Electrical Engineering, Technological Educational Institute of Crete, GR-71004 Heraklion, Crete, Greece (e-mail: fotis@staff.teicrete.gr)

${ }^{2}$ Solar Radiation Monitoring Laboratory, Department of Physics, 1274 University of Oregon, Eugene, Oregon 974031274, USA

(e-mail: fev@uoregon.edu)
}

(National Strategic Reference Framework 2007 - 2013). The authors would also to thank B. Marion for providing the NREL Test's facility data. The Eugene data used in this project was generated under the NREL subcontract number AFU-2-22072-1.

\section{Introduction}

The cumulative installed capacity of photovoltaic systems is growing continuously. According to the photovoltaic barometer [1] the installed photovoltaic power in the European Union reached 87 GWp by 2014. The worldwide PV power is estimated at $180 \mathrm{GWp}$ while the actually installed power in 2013 was 139 GWp (EPIA). Following medium case scenarios EPIA reports that around 8-9 GWp per year of new PV power systems could be installed in EU countries by 2018. While it is clear that there is a growing demand for PV system performance monitoring/recording, there is also a demand for models [2] that can more accurately estimate the expected power output with a few basic input parameters. In addition, a comparison between actual and expected power will aid the detection of possible problems during the operational life time of a photovoltaic system. In the approach used in this study, specific functions are utilized that describe the effects of air mass, angle of incidence, low irradiance performance and inverter performance. Seasonal effects due to dust can also be included. These functions can either be determined experimentally or adopt generic functions available in the literature if specific data are not available. A minimum number of input parameters was used to keep the approach as simple as possible while providing quality performance estimates. In section 2 we discuss the experimental tests performed to determine basic parameters of the model. In section 3 the experimental data used to validate the data are presented. Information about the module is also given. In section 4, we discuss the first results from the validation process. Preliminary data concerning the performance of the model are given. Finally, in section 5 we summarize the results of the current work and refer to the next steps of the analysis that has to be accomplished for a thorough investigation of the capabilities of the model. 


\section{Experimental tests used to develop the model}

The model adopts the known linear dependence of photovoltaic power on solar irradiance and cell temperature ([3] and references there in). The temperature coefficient of maximum power at STC conditions can be utilized from the manufacturer's data sheet or can be determined through actual measurements at high solar irradiances, e.g. above $700 \mathrm{~W} / \mathrm{m}^{2}$. Angle of incidence effects for a specific module are determined through measurements of the short circuit current, the incident beam and diffuse irradiance, and the temperature of the module [4]. The module initially is pointed towards the sun so that the angle of incidence (AOI) is zero. Subsequently, the module is rotated away from the sun to allow the AOI to reach values in excess of 80 degrees. These measurements were taken over a short period of time in which the incident solar radiation was changing slowly. This enables separation of AOI effects from airmass/spectral effects. Currently this model assumes that the response of a module to the beam and diffuse irradiances is the same.

The peak power of a photovoltaic module is given at Standard Test conditions (STC) and specifically at an air mass of 1.5 or a zenith angle of 48 degrees. However, this is rarely the case, especially in winter for typical northern latitudes. Electromagnetic radiation from the sun is not equally affected as the rays traverse varying amounts of the atmosphere from sunrise to solar noon and then to sunset. The more atmosphere traversed, the greater the amount of scattering. Rayleigh scattering, the dominant scattering mechanism under cloudless skies, displays a strong wavelength dependence affecting mainly UV/optical wavelengths. Consequently, module performance will also be affected and it is clear that thin film module technologies will be more strongly affected than mono or polycrystalline. Air mass effects are studied through measurements of the short circuit current, the global irradiance in the plane of the module and the temperature of the module. The module under study is placed on a two axis tracker and measurements start at high air masses and continue up to solar noon where the air mass is at its minimum [4]. The data are reduced to STC conditions by correcting for the temperature and specific solar irradiance recorded during the measurements. Such measurements are especially useful since photovoltaic modules can be used to assess the spectrally averaged solar resource incident on the module. Solar modules make bad pyranometers because it is difficult to separate the diffuse and beam irradiance components that have different spectral dependencies. In a first approach, we can examine the dependence of the produced DC power upon the airmass.

A photovoltaic module can be described by the one diode model which is characterized by a series and a shunt resistance. Typical values are less than $1 \mathrm{Ohm}$ and greater than $100 \mathrm{Ohm}$, respectively. At low light levels, e.g. below $300 \mathrm{~W} / \mathrm{m}^{2}$, the effect of the shunt resistance becomes important since the equivalent resistance of the module during operation approaches the value of the shunt resistance. At this stage the fractional loss of power due to the shunt resistance increases. In order to study the effect of the change of the efficiency of a module as a function of solar irradiance we employed stainless steel meshes of various transmittances. Use of such meshes allows one to vary the incoming solar irradiance by a factor of 10 , reaching irradiance levels well below 300 $\mathrm{W} / \mathrm{m}^{2}$. The module temperature is recorded along with the solar irradiance in front of and back of the mesh. The solar irradiance in front of the mesh is used to assess the stability of the ambient conditions.

\section{The validation data}

Once the model is setup, validation data are needed to check and verify the various components of the model. NREL conducted a field campaign at three different sites in the United States to study the performance of several PV modules at different climatic conditions [5]. Quality assessment methods were implemented by NREL to create a reliable data base that can be used to study the performance of PV modules and validate relevant models. Furthermore, daily, weekly, monthly maintenance

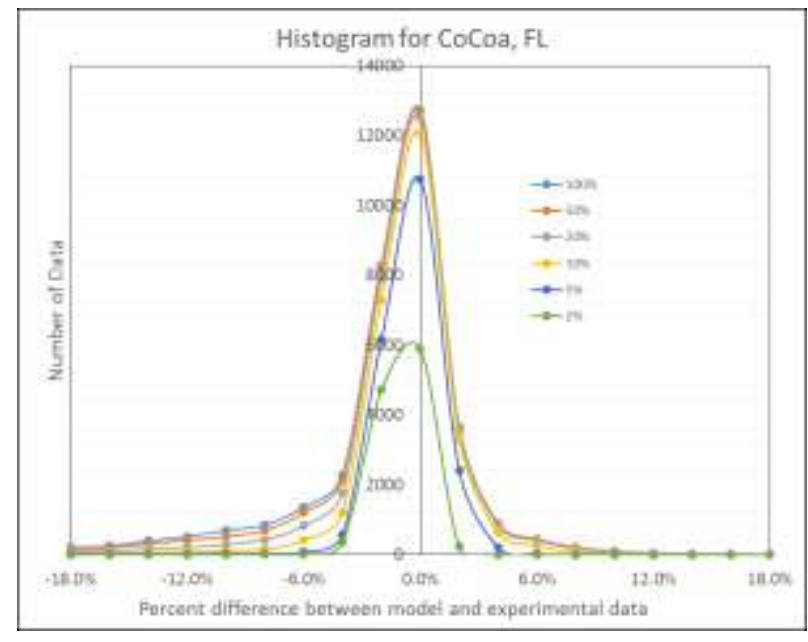


Fig. 1 The histogram of the percent difference between model and experimental data is shown for the selected uncertainties for Cocoa, FL.

along with the quality of the measurement equipment guaranteed low measurement errors.

In this work we adopt data acquired from a single multi-crystalline silicon module that was tested at two different sites for a total of more than two years. The module was first setup at a tilt of $28^{\circ} .5$ due south in Cocoa, FL (subtropical climate) for approximately fourteen months. During this time I-V traces were recorded every five minutes along with several environmental parameters. The same module was then setup at a tilt of $44^{\circ}$ due south at Eugene, OR (marine climate) for thirteen months. Before and after the experiment, NREL performed indoor performance measurements at Standard Test Conditions (STC) as an additional test of the integrity of the measurements. The adopted STC power used in the calculations is 45 Watts. It is clear that the two sites mentioned above offer an excellent tool to study the effects of diverse climates upon the performance of a module.

\section{Results-Discussion}

The model under validation has been presented in [3]. It is clear that the model can be improved if diffuse and beam solar irradiance data were separately available. However, since usually only plane of array (POA) irradiance data are available we proceed the analysis using a single irradiance value (global irradiance at POA).

The model can account for the effects of the propagation of sunlight through the atmosphere as described in [4] and [6]. This function is usually determined through measurements of the short circuit current and it can be tested against this data to check if the residuals are reduced. Another function used in the model refers to the effect caused by a nonzero angle of incidence of the incoming irradiance. If specific module data are not available a generic curve from modules of the same technology can be adopted ([4], [6]). The model also

Fig. 2 The histogram of the percent difference between model and experimental data is shown for the selected uncertainties for Eugene, OR.

takes into account the effect of the changing efficiency of a cell or module as the incoming solar irradiance drops below $400-500 \mathrm{~W} / \mathrm{m}^{2}$. It is clear that more functions can added if available. For example, seasonal dust effects can

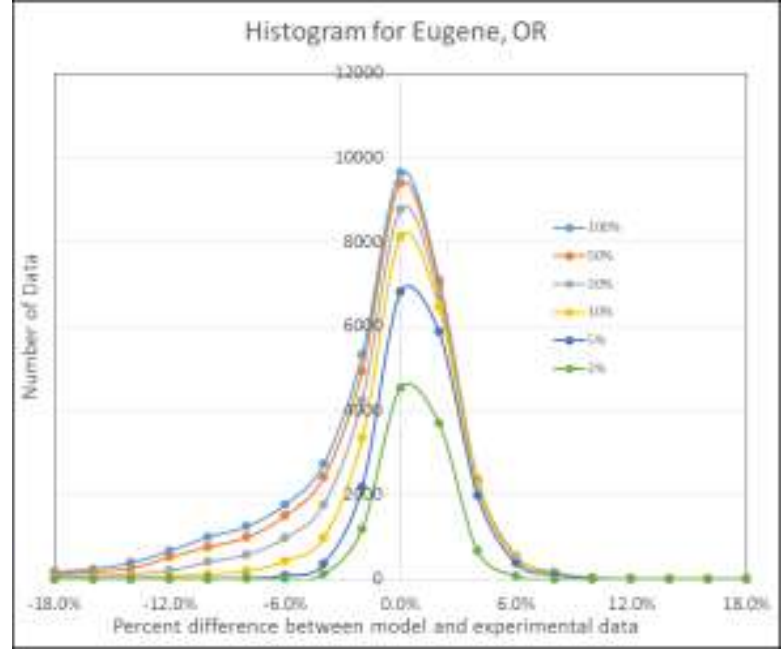

be incorporated to the model. The coefficient $\omega$ may be used to account for all other factors that cause loss of energy. This factor may also be used to account for the effect of aging of the modules. Currently this factor is set to 1 .

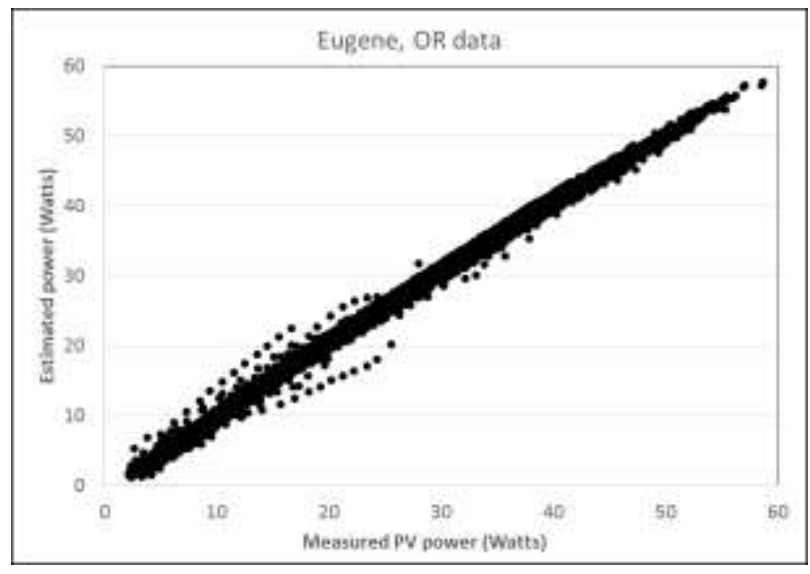

Fig. 3 A one to one plot for the PV power produced by a crystalline module in Eugene, OR. The y-axis shows the modelled power while the $\mathrm{x}$-axis shows the actually measured power.

Given the quality assessment performed by NREL, we selected the data according to the stated uncertainties given in the data files. The relative errors on the photovoltaic power and on the solar irradiance were chosen to be less than the limits listed in Table 1. We tested the model against sets of data satisfying relative errors in irradiance and power less than $100 \%, 50 \%, 20 \%$, $10 \%, 5 \%, 2 \%$. It is pointed out that uncertainties larger than $100 \%$ typically occur at solar irradiances less than 80 $\mathrm{W} / \mathrm{m}^{2}$ or maximum module power less than 2.5 Watts (around $6 \%$ of the module's peak STC power). Table 1 provides the number of data points used in the validation procedure as a function of the uncertainty in the plane of array irradiance and the DC power produced by the module. The percent difference between the model and the measured data was calculated as $\left(\mathrm{P}_{\text {model }}-\mathrm{P}_{\text {data }}\right) / \mathrm{P}_{\text {data }}$. Histograms were created and Gaussian profiles were used to fit them (Fig.1 for Eugene data). 
Table 1 No of Data

\begin{tabular}{|c|r|r|}
\hline & & \\
\hline $\begin{array}{l}\text { Relative } \\
\text { Error }\end{array}$ & Cocoa, FL & Eugene, OR \\
\hline $100 \%$ & 33597 & 34129 \\
\hline $50 \%$ & 32337 & 31646 \\
\hline $20 \%$ & 29605 & 27167 \\
\hline $10 \%$ & 25897 & 22698 \\
\hline $5 \%$ & 20405 & 17876 \\
\hline $2 \%$ & 11219 & 10276 \\
\hline
\end{tabular}

The FWHM for the different data sets selected according to the adopted uncertainties lie in the range of $2.6 \%$ (for the $<2 \%$ uncertainty data) to $3.7 \%$ (for the $<100 \%$ uncertainty data). Thus, at least with the current high quality data the typical uncertainty of the model is around $4 \%$ using the Cocoa, FL data. The asymmetry seen in the Gaussian profiles at negative values of the percentage difference and for data errors larger than around $10 \%$ is under investigation (Fig. 1).

Gaussian profiles were also fit to the data from Eugene, OR shown in Fig. 2. In this case the FWHM are found in the range of $3.8 \%$ (for the $<2 \%$ uncertainty data) to $5.4 \%$ (for the $100 \%$ uncertainty data). Thus, the typical uncertainty of the model is around $5 \%$. The asymmetry seen also in these Gaussian profiles at negative values of the percent difference and for data errors larger than around $10 \%$ is under investigation in an attempt to determine the factors that influence the shape of the distribution. Furthermore, the actually measured power and that predicted by the model are shown in Fig. 3 as a linear one to one plot. The data set in this plot originates from the test site at Eugene, OR and includes 31600 data points satisfying AOI angles less than $80^{\circ}$ and relative errors on the plane of array irradiance and measured DC power of less than $50 \%$.

In order to study the effect of the low irradiance losses we adopted a subset of the $20 \%$ uncertainty data from Cocoa, FL (Table 1). The additional filter that was applied involved angles of incidence less than 60 degrees to avoid angular effects that could affect the results. Two different data sets were created. The first included a generic correction for the low irradiance losses while in the second data set a constant efficiency was assumed for low and high irradiance values. The histogram (blue bars) in Fig. 4 was obtained when the low irradiance correction (LIR) correction was made. However, when the LIR correction is not included in the model, the histogram (orange bars) shows excess positive residuals. As the model predicts, the values are overestimated when the low irradiance level correction is absent. It should be possible to improve the fit if data specific to the module under study were available. However, a generic curve describing the performance of crystalline silicon modules under low light conditions can fit the experimental data quite well.

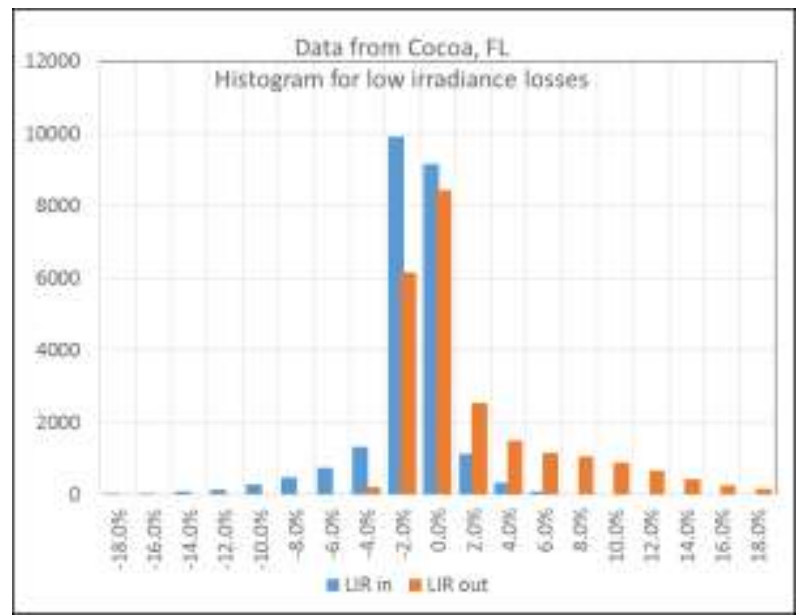

Fig. 4 The blue histogram results from data where a generic efficiency curve is included in the calculations. The orange histogram results when the low irradiance correction is absent from the model.

We also investigated the angular performance of the model. Using data from Cocoa, FL the model with and without the angle of incidence function was applied. The FWHM of the distribution increases if the correction is not applied and it becomes asymmetric around zero. In this case, the model overestimates the photovoltaic power and the number of positive residuals increases. The corresponding histograms are similar to those seen in Fig. 4 and for this reason they are not shown here.

The work being carried now involves use of all the data available to validate the model and study the residuals as a function of the various parameters like air mass, angle of incidence. The low irradiance performance of the model is also examined in relation to the data.

\section{Conclusion}

A simple model for the photovoltaic power is validated against high quality data. The data examined so far suggest that the corrections for angle of incidence and low irradiance effects provide a satisfactory representation of the data at a level of around $5 \%$. However, these are preliminary results and a better overview of its performance will be available when all 
available data are used in the validation process.

\section{References}

[1] EurObserv'ER barometer, 2014. http:www.eurobserv-er.org/

[2] Ding K., Ye Z. and Reindl T. "Comparison of parameterisation Models for the Estimation of the maximum power output of pv modules", Energy Procedia, 25, pp. 101-107, 2012

[3] Mavromatakis F., Makrides G., Georghiou G., Pothrakis A., Franghiadakis Y., Drakakis E., Koudoumas E., "Modelling the photovoltaic potential of a site", Renewable Energy, 35, pp13871390,2010
[4] D.L. King, J.A. Kratochvil and W.E. Boyson, "Measuring Solar Spectral and Angle-of-Incidence Effects on Photovoltaic Modules and Solar Irradiance Sensors", 26th IEEE Photovoltaic Specialists Conference, Spetember 29 - October 3, 1997, Anaheim, California, USA

[5] Marion W., Anderberg A., Deline C., Glick S., Muller M., Perrin G., Rodriguez J., Rummel S., Terwilliger K. and Silverman T.J. "User's manual for Data for validating models for pv module performance", National Renewable Energy Laboratoy, Technical Report, VTP-5200-61610, 2014

[6] Fanney A.H., Davis M.W., Dougherty B.P., King D.L., Boyson W.E. and Kratochvil J.A. "Comparison of Photovoltaic Module Performance Measurements", Transactions of the ASME, 128, pp. 152-160. 2006. 